\title{
A liquid metal-based triboelectric nanogenerator as stretchable electronics for safeguarding and self-powered mechanosensing
}

\author{
Wang Sheng, Xuan Shouhu and Gong Xinglong* \\ CAS Key Laboratory of Mechanical Behavior and Design of Materials, Department of Modern Mechanics, CAS Center for \\ Excellence in Complex System Mechanics, University of Science and Technology of China (USTC), Hefei 230027, China \\ *Corresponding author's email: gongxl@ustc.edu.cn
}

Introduction. Wearable electronics as one of the most promising technologies in next generation of artificial devices have achieved revolutionary progresses in the past decades ${ }^{(1)}$. They have potential applications in sensors, artificial skins, touch panel and smart textile ${ }^{(2)}$. However, external power sources are always required to drive these systems. An ideal strategy to overcome the inconvenience is developing novel electronics with self-powered property which can harvest energy and transform it to electric energy due to the universally available bio-mechanical energy from ambient environment. Since first reported in 2012, triboelectric nanogenerator (TENG) with high electronic output performance, easy fabrication and environmental friendliness proves its high efficiency in energyharvesting in recent years ${ }^{(3)}$. It can successfully convert mechanical energy into electric energy which can act as a new sustainable power source as well as self-powered sensor. However, most TENGs could not resist and harvest impact kinetic energy in daily life. Harsh interactions including friction force, compression and even violent collision often exist during exercise and work which may lead to property loss and personal injury. With the rise of personal security awareness nowadays, it is imperative to develop multifunctional stretchable TENG with safeguarding and force sensing performance to further diversify TENGs and satisfy actual demand of utilization.

In this report, a stretchable self-powered triboelectric nanogenerator with protecting and force-sensing properties has been designed by injecting GaInSn liquid metal into shear stiffening gel/ polydimethylsiloxane (SSG/PDMS) matrix. The storage modulus of SSG/PDMS is rate dependent which can change from $3.99 \mathrm{kPa}$ to $0.16 \mathrm{MPa}$ with the increase of shear frequency. Owing to the triboelectrification and electrostatic induction effect, the TENG device with optimized thickness of $4 \mathrm{~mm}$ shows high efficiency of gathering mechanical energy with current of $5.10 \mu \mathrm{A}$, and an output power of 182.17 $\mu \mathrm{W}$ (Fig. 1). Moreover, the as-prepared TENG exhibits enhanced triboelectric power of $323.97 \mu \mathrm{W}$ even stretched to $80 \%$. The nanogenerator acted as a selfpowered sensor also shows multiple sensing performance which can monitor various physiological excitations . Benefited from the shear stiffening effect, the TENG device can sustain and impede continuous collisions and decrease the impact force from 1687.37 $\mathrm{N}$ to $566.40 \mathrm{~N}$ as well as increasing the buffer time from 1.10 to $2.75 \mathrm{~ms}$ (Fig. 2). Thus, the reliable multifunction of the liquid metal-based TENG ensures its practical applications in wearable electronics, green energy sources and safeguards.

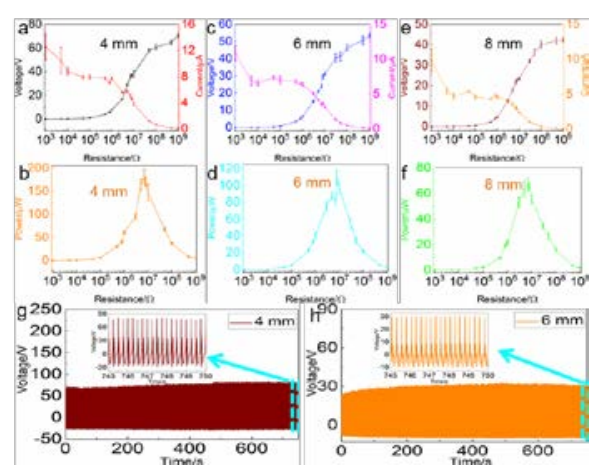

Fig. 1 Thickness dependent of TENG subjected to $27 \mathrm{~N}$ at a frequency of $10 \mathrm{~Hz}$ with different external resistance: electric voltage, current and power output of 4 (a, b), 6 (c, d) and 8 (e, f) mm; Stability of TENG with $4(\mathrm{~g})$ and $6(\mathrm{~h}) \mathrm{mm}$ at $500 \mathrm{M} \Omega$.

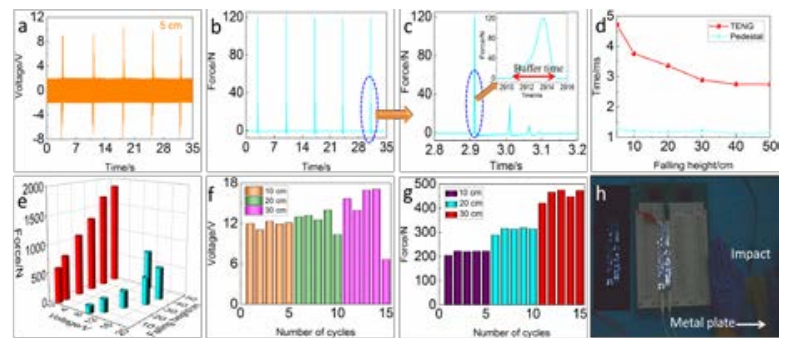

Fig. 2 Demonstration of GaInSn-based TENG as a selfpowered force sensor with safeguarding performance. Output voltage (a) and impact force (b) signals of TENG generated by the impact from $5 \mathrm{~cm}$, typical force-time curves impacted on TENG (c), metal force sensor (d) and the corresponding buffer times (e), voltage and impact force vs falling heights in the range of 5-50 cm (The red bars represent the signals loaded on force sensor) (f), cyclic stability of output voltage (g) and loading force (h) of TENG, power LEDs by impacting a stretchable TNEG (4 $\mathrm{mm})$ using metal plate (i).

\section{References.}

(1) Someya, T., Bao, Z. N., Malliaras, G. G., Nature, 540, (2016), 379-385.

(2) Chan, K. C., Hee, L. H., Hwan, O. K., Yun, S. J., Science, 535, (2016), 682-687.

(3) Fan, F. R., Tian, Z. Q., Wang, Z. L., Nano Energy 1, (2012), 328-334. 